\title{
COVID-19 serologic testing: FAQs and caveats
}

$\mathrm{T}$ HERE HAS BEEN an immense amount of discussion regarding the potential usefulness of serologic testing for COVID-19. Serologic testing has never been routinely used for diagnosing infections with "respiratory viruses" such as influenzae, parainfluenzae, respiratory syncytial viruses, adenoviruses, or metapneumovirus, nor was it used routinely for diagnosis during the global epidemics of severe acute respiratory syndrome (SARS), Middle East respiratory syndrome (MERS), and H1N1 influenza. However, the pandemic status of COVID-19 and the shortage of nucleic acid detection kits and swabs in certain areas raise the prospect of resorting to serology as an alternative to direct testing for the virus, and it is relevant to ask how useful it may be. The Infectious Diseases Society of America has recently issued a clear statement on $\mathrm{CO}$ VID-19 serology. ${ }^{1}$

The following addresses some common questions regarding serologic testing for $\mathrm{CO}$ VID-19.

\section{Is $\lg M / \lg A$ serology reliable for diagnosing acute symptomatic COVID-19?}

Based on recent publications, ${ }^{2}$ the appearance of detectable immunoglobulin M (IgM) antibodies after infection with COVID-19 is delayed, resulting in abysmal sensitivity ranging from $17 \%$ to $50 \%$ in the first 10 to 14 days after the "onset" of symptoms. Note that this is not days after exposure or infection, but rather days after the onset of clinical symptoms. Unfortunately, the results may not be clinically useful because COVID-19 often progresses very quickly within the first 7 to 10 days. ${ }^{3}$ Thus, by the time of seroconversion, patients could be critically ill with septic shock doi:10.3949/ccjm.87a.20054 or multiorgan failure, or they could die before seroconverting.

Most hospitalized patients typically receive the diagnosis of COVID-19 by nucleic acid testing before admission or up to 24 hours after admission. Unfortunately, by the time of serologic diagnosis, the patient may have inadvertently infected innumerable contacts.

There are no carefully peer-reviewed studies regarding the specificity of IgM and IgA tests, even though numerous point-of-care and nonpoint-of-care enzyme immunoassays (EIAs) are commercially available. IgM serologic tests, in general, have an inherent predisposition to false-positive results. Viruses as distantly related as Dengue virus have been reported to cause false-positive IgM results in COVID-19 pointof-care serologic tests. ${ }^{4}$

COVID-19 IgA EIAs had false-positive results in $20 \%$ of samples from 2,018 patients in the United States (author's personal communications). The potential for a rapidly progressing clinical course of COVID-19, combined with the low sensitivity of IgM testing during the first 10 days of clinical infection, makes this low specificity of IgA testing a concern, since class-switching to IgA typically occurs after the appearance of IgG.

\section{Is IgG serology a reliable option for diagnosing acute or convalescent COVID-19?}

$\mathrm{IgG}$ seroconversion is delayed after the onset of symptoms (more than 35 days in some cases), but typically occurs in 2 to 3 weeks, at which time it can be detected if the test specificity is high. Commercially available serologic assays, typically enzyme-linked immunosorbent assays (ELISAs), require validation with a plaquereduction neutralization test (PRNT).

In brief, PRNT requires mixing live viruses with serially diluted serum followed by
The pandemic

status

of COVID-19

and the

shortage

of supplies

raise

the possibility

of serologic

testing

as an

alternative

to direct

testing 
cell cultures to view cytopathic effect. PRNT is a functional assay that requires significant expertise and a biosafety level 3 facility (not available in hospitals), and it is not amenable to automation; however, it is necessary when any new assay is being validated. Ideally, this test should be done by manufacturers prior to US Food and Drug Administration submission; if this is a lab-developed test, the onus is on the lab to ensure PRNT is done on-site or in collaboration with a reference lab that has PRNT capability. Additionally, PRNT needs to be done head-to-head against other known coronaviruses, particularly those that are commonly acquired in the community (eg, 229E, OC43, NL63, HKU1), which have always been detected using nucleic acid amplification tests. Thus far, none of the published studies or commercially available kits have documentation of such validation.

That said, PRNT has its limitations. Previous exposure to common coronaviruses may lead to an early and high-titer humoral immune response to SARS-CoV-2. As time elapses, however, the humoral response probably becomes more specific to SARS-CoV-2. Studies have shown greater than $90 \%$ sero-

By the time of seroconversion, the patient could be critically ill or dead prevalence of common coronaviruses in the United Sates. Interestingly, Wölfel et $\mathrm{al}^{2}$ report finding a significant degree of serologic cross-reactivity between SARS-CoV-2 and common coronaviruses. Further, IgG responses were much stronger and appeared earlier than IgM responses. It seems that exposure to SARS-CoV-2 triggers previous memory response to all common coronaviruses. Based on the current information, it is not clear which target provides the best specificity, but specificity should increase over time as the immune response becomes more fine-tuned. This, however, will be well beyond the recovery time and, thus, of no use for diagnostic purposes.

In addition to cross-reactivity with common coronaviruses, false-positive results are seen using serum with elevated antinuclear antibody titers. Elevated titers are relatively common in patients over age 50, which happens to overlap with the median age for $\mathrm{CO}$ VID-19 diagnosis. False-positive results have also been documented with serum from patients with influenza or influenza vaccine re- cipients. Flu vaccine recipients constitute a large population- $45 \%$ of the adult US population, according to the US Centers for Disease Control and Prevention (CDC) - who may have overlapping signs and symptoms of influenza and COVID-19.

On the IgG side, false-positives using both EIA and point-of-care testing kits also have been observed in serum samples from patients with herpes simplex virus type 1 , human metapneumovirus, enterovirus, parvovirus B19, and sera-positive rheumatoid factor, among others. Finally, even if IgG is to be used with a highly specific ELISA for diagnosing acute COVID-19 infection, one still has to wait several weeks to see a minimum 4-fold rise in antibody levels. This would be too late to be of clinical use. And testing requires a minimum of 2 blood draws (acute and convalescent), exposing sick patients to even more healthcare environments.

\section{Is IgG serology reliable for evaluating infectivity and clinical immunity to reinfection with COVID-19?}

No one knows. Patients with a positive IgG result may still be sick and can shed the virus through their respiratory secretions or stools. SARS-CoV-2 is an enveloped RNA virus belonging to the Coronaviridae family, which includes common coronaviruses such as 229E, OC43, NL63, HKU1, and several that infect animals. Upper respiratory samples can remain positive for viral RNA for a few weeks after onset, when patients are supposed to have IgG antibodies. Viral shedding in stool has been reported for up to 47 days, which speaks against authentic neutralizing capacity of tissue-transudated $\operatorname{IgG}$ and secretory IgA antibodies. ${ }^{5}$ SARS-CoV (a SARS-CoV-2 sister virus) has been grown in cultures from upper respiratory samples in $54 \%$ of cases at 2 weeks after symptom onset and in 16\% of cases at 3 weeks after symptom onset, despite documented seroconversion in more than 92\% of patients assessed by PRNT that detected "neutralizing antibodies." ${ }^{6}$ Thus, having circulating neutralizing antibodies may not ensure lack of infectivity. This has yet to be shown in SARS-CoV-2.

As of this writing, the CDC has not established guidelines for occupational health isola- 
tion disposition based on serologic testing, other than using 2 consecutive negative nucleic-acid amplification tests at least 24 hours apart. ${ }^{?}$

Regarding COVID-19, the correlate of protection is not known, although these levels have been established for many other viral diseases. For example, the correlate of protection for hepatitis B is a surface antibody level at or very close to $10 \mathrm{mIU} / \mathrm{mL}$, and this measure is routinely used for occupational health purposes. For COVID-19, the correlate of protection has to be established in large, well-designed randomized controlled trials, which have not been conducted. Therefore, determination of "immune status" of individuals, including healthcare workers, to SARS-CoV-2 cannot be established at this time using serology. To further confound matters, all individuals can be infected and become sick with common coronaviruses in the community in almost every season and sometimes several times during a season. This suggests that immunity to some coronaviruses is short-lived, and lingering IgG antibodies from previous seasons does not mean an individual is necessarily immune to infection with the same coronaviruses. Furthermore, cell-mediated immunity (typically mediated through CD8+ memory $\mathrm{T}$ cells) also plays a role.

More recently, it has been shown that 20\% of individuals do not mount neutralizing antibodies and over 50\% mount only low titer neutralizing antibodies with geometric titer of 142 . The rest $(<30 \%)$ are able to mount hightiter neutralizing antibodies, but whether they will last and whether they are protective is not known. ${ }^{8}$

\section{Is IgG serology reliable for screening a COVID-19-convalescent donor?}

The discussions in the previous 2 items provide a segue to answer this question. First, we do not know if EIA results correlate well with PRNT (ie, ELISA antibodies vs neutralizing antibodies). And if they do correlate well, then second, we do not know if the socalled neutralizing antibodies are neutralizing enough to confer immunity.

Shen et $\mathrm{al}^{9}$ gave critically ill patients infusions of $400 \mathrm{~mL}$ of convalescent plasmas collected from donors with clinically resolved COVID-19. Interestingly, the critically ill recipients' pretransfusion neutralization titers were approximately only 1 dilution different than those of the donors (pretransfusion neutralizing antibody geometric titers of 192 and 80 , in donors and patients, respectively). Further, Duan et $\mathrm{a}^{10}$ found that severely ill patients had neutralization titers as high as 1:640 before receiving transfusions of convalescent plasma. Healthy and COVID-19-resolved donors had titers higher than 640.

These results raise the question as to why patients who already had mounted neutralizing antibody titers were still critically ill. This could be explained by the phenomenon called antibody-dependent enhancement, in which viruses can gain access to Fc gamma receptor-expressing cells via antibody-recognizing receptors as opposed to viral receptors and proliferate or trigger those cells to respond with a vigorous and potentially harmful cytokine release (cytokine storm). More recently, Wölfel et $\mathrm{al}^{2}$ grew SARS-CoV-2 in upper and lower respiratory samples from onset until day 8 but not beyond that. This suggests that transfusion of convalescencephase plasma may not have a role beyond day 8 after onset. This is important, as passive immunotherapy is typically considered in critically ill patients who are well beyond this time point.

Antibody-dependent enhancement has been shown in coronaviruses, which may potentially lead to more severe subsequent coronaviral diseases. Although this may have implications for vaccine design (similar to those of Dengue vaccine), it may also lead to potential adverse outcomes for convalescent plasma therapy. At this juncture, we do not have any evidence that plasma from patients who have recovered offers clear clinical benefit, as it showed mixed results for SARS or MERS. ${ }^{11}$ Further, SARS-CoV and SARS-CoV-2 can cause syncytium formation among lung epithelial cells, thereby paving the way for cell-to-cell transmission of the virions. In this way, virions may be protected from antibody neutralization.

Using a serologic test with poor or unknown performance characteristics to "greenlight" distributing blood products (plasma) is not really an undertaking for hospital labs. The

\section{Patients with a positive lgG may still be sick and can shed the virus}


US Food and Drug Administration (FDA), ${ }^{12}$ however, recommends neutralizing antibody titers of at least 1:160, but a titer of 1:80 may be considered acceptable if an alternative matched unit is not available. The FDA also recommends that convalescent plasma be considered only for patients with severe or immediately life-threatening COVID-19. The FDA further clarifies that, although promising, convalescent plasma has not yet been shown to be effective in every disease studied. It is therefore important to determine through clinical trials, before routinely administering convalescent plasma to patients with COVID-19, that it is safe and effective to do so. ${ }^{12}$

In short, at this point, using serology to screen COVID-19-convalescent donors is fraught with risk, not only because there is no robust science to back it up, but also because there are no FDAapproved products for donor screening. Further, the correlation between neutralization assays and other test formats has been poor, ${ }^{2}$ making it hard to use commercial assays for this purpose.

\section{Is IgG serology reliable for SARS-CoV-2 serosurveys?}

Maybe, but it very much depends on the specificity of the assay. Serology may only be good for surveillance or seroepidemiologic studies, which is a public health function or an academic project. Once fine-tuned assays are available and resources allow, impact assessments will need to be done on a large scale in collaborative studies performed using well-balanced and unbiased samples that include multiple age, sex, and geographic cohorts.

Another aspect here is to assess what percentage of infected individuals remain asymptomatic and to calculate the case-fatality rate (CFR). The former is helpful as background epidemiologic knowledge, but the CFR is even more important, although it comes at a potential cost. The cost is that taking asymptomatic cases into account when the CFR is calculated amid an outbreak, a sudden drop in the publicly announced CFR would potentially lead to loosening precautionary measures such as social distancing by the general public, which may lead to further spread of the infection.

Here's some math to consider. As of May 13,2020 , there were $1,420,581$ cases in the United States. Also, let's say only $20 \%$ of cases become severely symptomatic, which is a gross underestimation because according to the Morbidity and Mortality Weekly Report, $87 \%$ will be symptomatic. ${ }^{13}$ Thus, at most, we'll have a total of 7,102,905 infected cases in the United States so far. Given the 2019 US population of 328.2 million, this means a seroprevalence of $2.16 \%$. An ideal IgG kit (for serosurveys) with a sensitivity of $100 \%$ and specificity of $95 \%$, used in a context of a pretest probability of $2.16 \%$, would give us a positive predictive value (PPV) of $30 \%$ (best-case scenario). Although a very big claim to make, even a specificity of $99 \%$ would yield a PPV of $69 \%$, meaning out of 100 positive IgG results, between 31 and 70 of them are more likely false-positive. This is of course for the whole country; a state such as New York can immensely skew the calculations, given the disproportionately high number of cases.

The seroprevalence in the State of Ohio is estimated as about $1.06 \%$ (based on published state government data as of May 13, 2020). Therefore, the PPV will be $17 \%$, based on specificity of $95 \%$, and $51 \%$ based on a specificity of $99 \%$. Again, all of these are calculated very generously.

Let's take a recent example from the media on coronavirus infections in Northern California. ${ }^{14}$ This study by Stanford University researchers suggested a seroprevalence of $2.5 \%$ to $4.2 \%$ in Santa Clara County. According to the above calculations and based on the premise for the test performance (sensitivity 100\%, specificity 95\%), the PPV for the California study would be $33 \%$ to $46 \%$, which is translated to a large false alarm. Another way to look at this is to compare their claim with peerreviewed literature. According to this serosurvey, the actual numbers of cases is estimated to be 50 to 85 times higher than what the county has announced. This roughly means that only $1.17 \%$ to $2 \%$ of infected individuals become symptomatic.

On a related note, according to a CDC study from the state of Washington, $87 \%$ of coronavirus-infected individuals became symptomatic. ${ }^{13}$ This finding (87\%) is in sharp contrast with the results of the Stanford University serosurvey estimates. A subsequent study by Arons et $\mathrm{al}^{15}$ reported that $94 \%$ of residents of a skilled nursing facility with confirmed sta- 
tus became symptomatic. More recently, the CDC reported that of 4,336 exposed healthcare workers (median age 42) with confirmed COVID-19 diagnosis (by RNA testing), only $8 \%$ did not report any symptoms. ${ }^{16}$ It should also be mentioned that according to an earlier study, also from California, about $5 \%$ of individuals with flulike illness tested positive for COVID-19 by RNA testing. ${ }^{17}$

Serosurveys may help with understanding herd immunity. With a minimum calculated basic reproduction number of 2.2, a minimum of $55 \%$ of the population is needed to be immune to prevent large outbreaks. With current interventions we may never reach such a point unless an effective vaccine becomes available. Therefore, a serosurvey may not necessarily help with this aspect.

Finally, according to a large epidemiologic joint report from China and the World Health Organization, ${ }^{18}$ only $1 \%$ of cases were asymptomatic based on typical symptoms; of symptomatic cases, $81 \%$ were mild or moderate and $19 \%$ were severe or critical. This was also reviewed and summarized later by $\mathrm{Wu}$ and McGoogan ${ }^{19}$; although in almost all jurisdictions severe and critical cases get tested for RNA (as are less-severe cases based on expanded other indications), it is relatively safe to multiply the announced number of confirmed cases by 5 to arrive at the estimated total number.

All in all, a careful analysis of harm and cost vs benefit needs to be done prior to conducting such large-scale serosurveys, if needed at all.

\section{REFERENCES}

1. Infectious Diseases Society of America. IDSA COVID-19 antibody testing primer. Updated April 29, 2020. Accessed May 1, 2020. https://www.idsociety.org/globalassets/idsa/public-health/covid-19/ idsa-covid-19-antibody-testing-primer.pdf

2. Wölfel R, Corman VM, Guggemos W, et al. Virological assessment of hospitalized patients with COVID-2019. Nature 2020 April 1. doi:10.1038/s41586-020-2196-X

3. Bhatraju PK, Ghassemieh BJ, Nichols M, et al. Covid-19 in critically ill patients in the Seattle region - case series. N Engl J Med 2020 March 30. doi:10.1056/NEJMoa2004500

4. Yan G, Lee CK, Lam LTM, et al. Covert COVID-19 and false-positive dengue serology in Singapore. Lancet Infect Dis 2020 March 4. doi:10.1016/S1473-3099(20)30158-4

5. Centers for Disease Control and Prevention. Coronavirus Disease 2019 (COVID-19). April 16, 2020. Accessed May 1, 2020. https://www. cdc.gov/coronavirus/2019-ncov/hcp/faq.html

6. Chan KH, Poon LL, Cheng VC, et al. Detection of SARS coronavirus in patients with suspected SARS. Emerg Infect Dis 2004; 10(2):294-299. doi:10.3201/eid1002.030610

7. Centers for Disease Control and Prevention. Discontinuation of transmission-based precautions and disposition of patients with COVID-19 in healthcare settings (interim guidance). March 3, 2020. Accessed May 1, 2020. https://www.cdc.gov/coronavirus/2019-ncov/ hcp/disposition-hospitalized-patients.html

8. Hachim A, Kavian N, Cohen CA, et al. Beyond the spike: identification of viral targets of the antibody response to SARS-CoV-2 in COVID-19 patients. medRxiv May 2, 2020. doi:10.1101/2020.04.30.20085670

9. Shen C, Wang Z, Zhao F, et al. Treatment of 5 critically ill patients with COVID-19 with convalescent plasma. JAMA 2020; 323(16):15821589. doi:10.1001/jama.2020.4783

10. Duan K, Liu B, Li C, et al. Effectiveness of convalescent plasma therapy in severe COVID-19 patients. Proc Natl Acad Sci U S A 2020; 117(17):9490-9496. doi:10.1073/pnas.2004168117

11. Stockman L, Bellamy R, Garner P. SARS: systematic review of treatment effects. PLoS Med 2006; 3(9):e343.

doi:10.1371/journal.pmed.0030343
12. US Food and Drug Administration. Recommendations for investigational COVID-19 convalescent plasma. April 13, 2020. Accessed May 1, 2020. https://www.fda.gov/vaccines-blood-biologics/ investigational-new-drug-ind-or-device-exemption-ide-process-cber/ investigational-covid-19-convalescent-plasma-emergency-inds

13. Kimball A, Hatfield KM, Arons M, et al. Asymptomatic and presymptomatic SARS-CoV-2 infections in residents of a long-term care skilled nursing facility - King County, Washington, March 2020. MMWR Morb Mortal Wkly Rep 2020; 69(13):377-381. doi:10.15585/mmwr.mm6913e1

14. Mason M, Netburn D. Coronavirus infections could be much more widespread than believed, California study suggests. Los Angeles Times April 17, 2020. Accessed May 1, 2020. https://www.latimes.com/california/story/2020-04-17/coronavirus-antibodies-study-santa-clara-county

15. Arons MM, Hatfield KM, Reddy SC, et al. Presymptomatic SARSCoV-2 infections and transmission in a skilled nursing facility. $\mathrm{N}$ Engl J Med 2020 Apr 24 [Epub ahead of print]. doi:10.1056/NEJMoa2008457

16. CDC COVID-19 Response Team. Characteristics of health care personnel with COVID-19 - United States, February 12-April 9, 2020. MMWR Morb Mortal Wkly Rep 2020; 69(15):477-481. doi:10.15585/mmwr.mm6915e6

17. Spellberg B, Haddix M, Lee R, et al. Community prevalence of SARSCoV-2 among patients with influenzalike illnesses presenting to a Los Angeles Medical Center in March 2020. JAMA 2020 [Epub ahead of print]. doi:10.1001/jama.2020.4958

18. World Health Organization. Report of the WHO-China joint mission on coronavirus disease 2019 (COVID-19). Accessed May 17, 2020. https://www.who.int/docs/default-source/coronaviruse/who-chinajoint-mission-on-covid-19-final-report.pdf

19. Wu ZW, McGoogan JM. Characteristics of and important lessons from the coronavirus disease 2019 (COVID-19) outbreak in China. Summary of a report of 72314 cases from the Chinese Center for Disease Control and Prevention. JAMA 2020; 323(13):1239-1242. doi:10.1001/jama.2020.2648

Address: Kamran Kadkhoda, PhD, Department of Laboratory Medicine, LL3-3, Cleveland Clinic, 9500 Euclid Avenue, Cleveland, OH 44195; kadkhok@ccf.org 